PROCEEDINGS OF THE

AMERICAN MATHEMATICAL SOCIETY

Volume 136, Number 9, September 2008, Pages 3281-3291

S 0002-9939(08)09424-0

Article electronically published on May 1, 2008

\title{
ON STRICT INCLUSIONS IN HIERARCHIES OF CONVEX BODIES
}

\author{
VLADYSLAV YASKIN
}

(Communicated by N. Tomczak-Jaegermann)

\begin{abstract}
Let $\mathcal{I}_{k}$ be the class of convex $k$-intersection bodies in $\mathbb{R}^{n}$ (in the sense of Koldobsky) and $\mathcal{I}_{k}^{m}$ be the class of convex origin-symmetric bodies all of whose $m$-dimensional central sections are $k$-intersection bodies. We show that 1) $\mathcal{I}_{k}^{m} \not \subset \mathcal{I}_{k}^{m+1}, k+3 \leq m<n$, and 2) $\mathcal{I}_{l} \not \subset \mathcal{I}_{k}, 1 \leq k<l<n-3$.
\end{abstract}

\section{INTRODUCTION}

Let $K$ and $L$ be origin-symmetric star bodies in $\mathbb{R}^{n}$. Following Lutwak $[\mathrm{L}$ we say that $K$ is the intersection body of $L$ if the radius of $K$ in every direction is equal to the volume of the central hyperplane section of $L$ perpendicular to this direction, i.e. for every $\xi \in S^{n-1}$,

$$
\rho_{K}(\xi)=\operatorname{vol}_{n-1}\left(L \cap \xi^{\perp}\right) .
$$

The closure in the radial metric of the class of intersection bodies of star bodies gives the class of intersection bodies.

A generalization of the concept of an intersection body was introduced by Koldobsky in $\mathrm{K} 3$. Let $1 \leq k<n$ and let $K$ and $L$ be origin-symmetric star bodies in $\mathbb{R}^{n}$. We say that $K$ is a $k$-intersection body of $L$ if for every $(n-k)$-dimensional subspace $H \subset \mathbb{R}^{n}$

$$
\operatorname{Vol}_{k}\left(K \cap H^{\perp}\right)=\operatorname{Vol}_{n-k}(L \cap H) .
$$

The closure in the radial metric gives the class of $k$-intersection bodies, which will be denoted by $\mathcal{I}_{k}$. Note that $\mathcal{I}_{1}$ is the class of intersection bodies.

Koldobsky [K2] introduced the concept of embedding of a normed spaces in $L_{p}$, $p<0$, and in $\mathrm{K} 3$. he proved that $k$-intersection bodies are the unit balls of spaces that embed in $L_{-k}$.

A well-known property of $L_{p}$-spaces, proved in [BDK], is that, for any $0<p<$ $q \leq 2$, the space $L_{q}$ embeds isometrically in $L_{p}$, so $L_{p}$-spaces become larger when $p$ decreases from 2. Koldobsky [K2] extended this result to negative $p$ : Every $n$-dimensional subspace of $L_{q}, 0<q \leq 2$, embeds in $L_{p}$ for every $-n<p<0$.

However, it is an open problem as to whether a normed space $X=\left(\mathbb{R}^{n},\|\cdot\|\right)$ being embedded in $L_{-p}$ for some $0<p<n-3$ implies that $X$ embeds in $L_{-q}$ for

Received by the editors July 10, 2007.

2000 Mathematics Subject Classification. Primary 52A20, 52A21, 46 B04.

The author was supported in part by the European Network PHD, FP6 Marie Curie Actions, RTN, Contract MCRN-511953. Part of this work was done when the author was visiting Université de Marne-la-Vallée.

(C)2008 American Mathematical Society Reverts to public domain 28 years from publication 
all $p<q<n$. In particular, is it true that every $k$-intersection body is also an $m$-intersection body for $1<k<m<n-3$ ? Note that in some cases the above statement is known to be true. Since the product of positive definite distributions is also positive definite, one immediately obtains that if $X$ embeds in $L_{-p}, 0<p<n$, and $p$ divides $q, p<q<n$, then $X$ also embeds in $L_{-q}$; see [M1.

Related to these are the questions of showing that these classes of bodies are different for different values of $p$ and $q$.

It was shown by Koldobsky $\mathrm{K} 1$ that there is an $n$-dimensional $(n \geq 3)$ Banach subspace of $L_{1 / 2}$ that does not embed in $L_{1}$ (also a subspace of $L_{1 / 4}$ that does not embed in $\left.L_{1 / 2}\right)$. Later Borwein and his colleagues at the Center for Computational Mathematics at Simon Fraser University showed (by computer methods) that there is a Banach space that embeds in $L_{a / 64}$ but not in $L_{(a+1) / 64}$ for $a=1,2, \ldots, 63$. Another construction was given by Kalton and Koldobsky in [KK] and it allowed them to extend these results to all $0<p<q \leq 1$. Schlieper [S] used the construction from [K1 to show that there is a normed space that embeds in $L_{-4}$ but does not embed in $L_{-2}$, and also a normed space that embeds in $L_{-1 / 3}$ but not in $L_{-1 / 6}$.

In this paper we extend Schlieper's result to arbitrary integers, namely, we construct examples of origin-symmetric convex bodies which are $k$-intersection bodies, but not $l$-intersection bodies for $1 \leq l<k<n-3$. We should remark that all origin-symmetric convex bodies are $k$-intersection bodies for $k=n-1, n-2$ and $n-3 ;$ see [K4, p. 78].

Another result that we present here is motivated by papers of Weil [W], Neyman $\left[\mathrm{N}\right.$ and Yaskina [Y]. Weil constructed a convex body in $\mathbb{R}^{n}(n \geq 3)$ that is not a zonoid but all its projections onto hyperplanes are zonoids. Neyman showed that there are $n$-dimensional normed spaces that do not embed in $L_{p}$, but all their $(n-1)$-dimensional subspaces embed in $L_{p}$ for $p>0$. Yaskina constructed a body in $\mathbb{R}^{n}(n \geq 5)$ which is not an intersection body, but all of its central hyperplane sections are intersection bodies. (Note that all central sections of an intersection body are necessarily intersection bodies; see Fallert, Goodey and Weil [FGW]).

Here we generalize Yaskina's construction to prove the following. Let $\mathcal{I}_{k}^{m}$ be the class of convex bodies all of whose $m$-dimensional central sections are $k$-intersection bodies. There exists an origin-symmetric convex body $K \subset \mathbb{R}^{n}$, such that $K \in \mathcal{I}_{k}^{m}$ but $K \notin \mathcal{I}_{k}^{m+1}, k+3 \leq m<n$. One should compare this result with the fact (see e.g. [M1]) that all central sections of a $k$-intersection body are also $k$-intersection bodies provided that the dimension of the sections is greater than $k$. Therefore, $\mathcal{I}_{k}^{m+1} \subset \mathcal{I}_{k}^{m}$.

Finally let us remark that another generalization of intersection bodies was introduced by Zhang [Z]. These are called generalized $k$-intersection bodies by Koldobsky and $k$-Busemann-Petty bodies by E. Milman. See [K3], K4, Section 4.5], [M1, and [M2] for many interesting results explaining the relation between these different generalizations and their connection to the lower-dimensional Busemann-Petty problem.

$$
\text { 2. } \mathcal{I}_{k}^{m} \not \subset \mathcal{I}_{k}^{m+1}
$$

Let us start with the following criterion for $k$-intersection bodies.

Theorem 2.1 (Koldobsky [K3]). Let $K$ be an origin-symmetric star body in $\mathbb{R}^{n}$, $1 \leq k<n . K$ is a $k$-intersection body if and only if the Fourier transform of $\|x\|_{K}^{-k}$ is a positive distribution. 
The main result of this section is the following theorem.

Theorem 2.2. Let $k+3 \leq m<n$. There exists an origin-symmetric convex body $K$ that belongs to $\mathcal{I}_{k}^{m}$, but not to $\mathcal{I}_{k}^{m+1}$.

Proof. For a small $\epsilon>0$ define a body $K$ by

$$
\|x\|_{K}^{-k}=|x|_{2}^{-k}-2 \epsilon^{m-k}\|x\|_{E}^{-k}, \quad x \in \mathbb{R}^{n} \backslash\{0\},
$$

where $|x|_{2}$ is the Euclidean norm and $E$ is the ellipsoid given by

$$
\|x\|_{E}=\left(x_{1}^{2}+\cdots+x_{m}^{2}+\frac{x_{m+1}^{2}+\cdots+x_{n}^{2}}{\epsilon^{2}}\right)^{1 / 2} .
$$

Since $\|x\|_{E}^{-1} \leq|x|_{2}^{-1}$, we have that $\|x\|_{K}^{-1}$ is positive for a small $\varepsilon$, and so the body $K$ is well defined.

The proof of the theorem follows from the following three lemmas.

Lemma 2.3. The body $K$ is convex for small enough $\varepsilon$.

Proof. This is a standard perturbation argument; cf. [K4, p.96]. By construction, the body $K$ is obtained by perturbing the Euclidean ball. Since the latter has strictly positive curvature, it is enough to control the first and second derivatives of the function $\epsilon^{m-k}\|x\|_{E}^{-k}$. One can see that their order is $O\left(\epsilon^{m-k-2}\right)$, which is small for small enough $\epsilon$. Therefore $K$ also has positive curvature.

Recall that the Fourier transform of $|x|_{2}^{-k}, 0<k<n$, equals (see [GS, p. 363])

$$
\left(|x|_{2}^{-k}\right)^{\wedge}(y)=C_{n, k}|y|_{2}^{-n+k}
$$

where

$$
C_{n, k}=\frac{2^{n-k} \pi^{n / 2} \Gamma((n-k) / 2)}{\Gamma(k / 2)} .
$$

In order to compute the Fourier transform for the norms of ellipsoids, note that if $T$ is an invertible linear transformation on $\mathbb{R}^{n}$, then

$$
\left(|T x|_{2}^{-k}\right)^{\wedge}(y)=C_{n, k}|\operatorname{det} T|^{-1}\left|\left(T^{*}\right)^{-1} y\right|_{2}^{-n+k} .
$$

Lemma 2.4. For every $m$-dimensional subspace $H$ of $\mathbb{R}^{n}$, the body $K \cap H$ is a $k$-intersection body.

Proof. We have

$$
\|x\|_{K \cap H}^{-k}=|x|_{B_{2} \cap H}^{-k}-2 \epsilon^{m-k}\|x\|_{E \cap H}^{-k} .
$$

Since $E$ is an ellipsoid with semiaxes $\epsilon$ and $1, E \cap H$ is also an ellipsoid with semiaxes $a_{1}, \ldots, a_{m}$ such that $\epsilon \leq a_{i} \leq 1, \forall i=1, \ldots, m$. There is a coordinate system in $H$ such that

$$
\|y\|_{K \cap H}^{-k}=\left(y_{1}^{2}+\cdots+y_{m}^{2}\right)^{-k / 2}-2 \epsilon^{m-k}\left(\frac{y_{1}^{2}}{a_{1}^{2}}+\cdots+\frac{y_{m}^{2}}{a_{m}^{2}}\right)^{-k / 2} .
$$

Taking the Fourier transform of $\|y\|_{K \cap H}^{-k}$ in the plane $H$ we get

$$
\left(\|y\|_{K \cap H}^{-k}\right)^{\wedge}(\xi)=C_{m, k}\left(|\xi|_{2}^{-m+k}-2 \epsilon^{m-k} \prod_{i=1}^{m} a_{i} \cdot\left(a_{1}^{2} \xi_{1}^{2}+\cdots+a_{m}^{2} \xi_{m}^{2}\right)^{(-m+k) / 2}\right) .
$$


Let $a_{j}$ be the smallest semiaxis. Then for some $\lambda \geq 1$ we have $a_{j}=\lambda \epsilon$. Therefore,

$$
\prod_{i=1}^{m} a_{i} \leq \lambda \epsilon
$$

On the other hand if $\xi \in S^{m-1} \subset H$, then

$$
\left(a_{1}^{2} \xi_{1}^{2}+\cdots+a_{m}^{2} \xi_{m}^{2}\right)^{(-m+k) / 2} \leq a_{j}^{-m+k}=(\lambda \epsilon)^{-m+k} .
$$

Therefore,

$$
2 \epsilon^{m-k} \prod_{i=1}^{m} a_{i} \cdot\left(a_{1}^{2} \xi_{1}^{2}+\cdots+a_{m}^{2} \xi_{m}^{2}\right)^{(-m+k) / 2} \leq 2 \epsilon^{m-k} \lambda \epsilon(\lambda \epsilon)^{-m+k} \leq 2 \epsilon .
$$

So, if $\epsilon \leq 1 / 2$, then $\left(\|y\|_{K \cap H}^{-k}\right)^{\wedge}(\xi) \geq 0$ for all $\xi \in S^{n-1} \cap H$ and all $H$. Therefore all $m$-dimensional sections of $K$ are $k$-intersection bodies.

Lemma 2.5. There exists an $(m+1)$-dimensional section of $K$ which is not a $k$-intersection body.

Proof. Let $H=\left\{x \in \mathbb{R}^{n}: x_{m+2}=\cdots=x_{n}=0\right\}$. Then

$$
\|x\|_{K \cap H}^{-k}=\left(x_{1}^{2}+\cdots+x_{m+1}^{2}\right)^{-k / 2}-2 \epsilon^{m-k}\left(x_{1}^{2}+\cdots+x_{m}^{2}+\frac{x_{m+1}^{2}}{\epsilon^{2}}\right)^{-k / 2} .
$$

The Fourier transform in the variables $x_{1}, \ldots, x_{m+1}$ equals

$$
\begin{aligned}
\left(\|x\|_{K \cap H}^{-k}\right)^{\wedge}(\xi) & =C_{m+1, k}\left(\left(\xi_{1}^{2}+\cdots+\xi_{m+1}^{2}\right)^{(-m+k-1) / 2}\right. \\
& \left.-2 \epsilon^{m-k} \epsilon\left(\xi_{1}^{2}+\cdots+\xi_{m}^{2}+\epsilon^{2} \xi_{m+1}^{2}\right)^{(-m+k-1) / 2}\right) .
\end{aligned}
$$

If $\xi=(0, \ldots, 0,1) \in S^{m} \subset H$, then

$$
\left(\|x\|_{K \cap H}^{-k}\right)^{\wedge}(\xi)=C_{m+1, k}\left(1-2 \epsilon^{m-k} \epsilon \epsilon^{-m+k-1}\right)=-C_{m+1, k}<0 .
$$

Therefore $K \cap H$ is not a $k$-intersection body.

$$
\text { 3. } \mathcal{I}_{l} \not \subset \mathcal{I}_{k}, l>k
$$

We will need a few auxiliary lemmas.

Lemma 3.1. Let $k \in \mathbb{N} \cup\{0\}$ and $f \in C^{\infty}\left(S^{n-1}\right), f$ being even. Let $x=(r, \theta)$ be polar coordinates in $\mathbb{R}^{n}$, so that $f(\theta) r^{-p}=f\left(\frac{x}{|x|_{2}}\right)|x|_{2}^{-p}$. Then the Fourier transform of the distribution $f(\theta) r^{-p}, 0<p<n$, is a homogeneous degree $-n+p$ continuous on $\mathbb{R}^{n} \backslash\{0\}$ function, whose values on the unit sphere can be computed as follows:

(i) If $q<2 k, q$ is not an odd integer, then $\forall x \in S^{n-1}$,

$$
\begin{gathered}
\left(f(\theta) r^{-n+q+1}\right)^{\wedge}(x)=\frac{(-1)^{k+1} \pi}{2 \Gamma(2 k-q) \sin (\pi(2 k-q-1) / 2)} \\
\quad \times \int_{S^{n-1}}|(x, \xi)|^{2 k-q-1} \Delta^{k}\left(f(\theta) r^{-n+q+1}\right)(\xi) d \xi,
\end{gathered}
$$

where $\Delta$ is the Laplace operator on $\mathbb{R}^{n}$. 
(ii) If $q$ is an even integer, $q=2 k$, then $\forall x \in S^{n-1}$,

$$
\left.\left(f(\theta) r^{-n+2 k+1}\right)^{\wedge}(x)=(-1)^{k} \pi \int_{S^{n-1} \cap x^{\perp}} \Delta^{k}\left(f(\theta) r^{-n+2 k+1}\right)\right)(\xi) d \xi .
$$

(iii) If $q$ is an odd integer, $q=2 k-1 \geq 1$, then $\forall x \in S^{n-1}$,

$$
\begin{aligned}
\left(f(\theta) r^{-n+2 k}\right)^{\wedge}(x) & =(-1)^{k} \int_{S^{n-1}} \ln |(x, \xi)| \Delta^{k}\left(f(\theta) r^{-n+2 k}\right)(\xi) d \xi \\
& +(-1)^{k}(2-n) \int_{S^{n-1}} \Delta^{k-1}\left(f(\theta) r^{-n+2 k}\right)(\xi) d \xi
\end{aligned}
$$

Proof. (i) and (ii) are proved in [K4, Lemma 3.16].

(iii) is essentially from [Y, Lemma 2.4]. For completeness we include a proof. For $q$ close to 1 we use part (i) with $k=1$ to get

$$
\left(f(\theta) r^{-n+q+1}\right)^{\wedge}(x)=\frac{\pi \int_{S^{n-1}}|(x, \xi)|^{1-q} \Delta\left(f(\theta) r^{-n+q+1}\right)(\xi) d \xi}{2 \Gamma(2-q) \sin \frac{\pi(1-q)}{2}} .
$$

When $q$ approaches 1 , both the numerator and denominator in the right hand side tend to zero. Indeed, let us show that the limit of the numerator is zero:

$$
\lim _{q \rightarrow 1} \int_{S^{n-1}}|(x, \xi)|^{1-q} \Delta\left(f(\theta) r^{-n+q+1}\right)(\xi) d \xi=\int_{S^{n-1}} \Delta\left(f(\theta) r^{-n+2}\right)(\xi) d \xi
$$

Recall the relation between the spherical Laplacian $\Delta_{S}$ and Euclidean Laplacian $\Delta$ (see e.g. [G, p. 7]). If $f$ is a homogeneous function of degree $m$, then on the sphere

$$
\Delta_{S} f=\Delta f-m(m+n-2) f
$$

Since $f(\theta) r^{-n+2}$ is a homogeneous function of degree $-n+2$, the previous formula implies $\Delta\left(f(\theta) r^{-n+2}\right)(\xi)=\Delta_{S}\left(f(\theta) r^{-n+2}\right)(\xi)$. Due to the fact that $\Delta_{S}$ is a selfadjoint operator, [G, p. 7], we have

$$
\int_{S^{n-1}} \Delta_{S}\left(f(\theta) r^{-n+2}\right)(\xi) d \xi=0 .
$$

In order to compute the limit of (11) as $q \rightarrow 0$, apply l'Hopital's rule:

$$
\begin{aligned}
\left(f(\theta) r^{-n+2}\right)^{\wedge}(x)= & -\int_{S^{n-1}} \ln |(x, \xi)| \Delta\left(f(\theta) r^{-n+2}\right)(\xi) d \xi \\
& -\int_{S^{n-1}} \Delta\left(f(\theta) r^{-n+2} \ln r\right)(\xi) d \xi
\end{aligned}
$$

Computing the Laplacian in the latter integral and using Euler's formula for derivatives of homogeneous functions, we get

$$
\begin{aligned}
\left(f(\theta) r^{-n+2}\right)^{\wedge}(x)= & -\int_{S^{n-1}} \ln |(x, \xi)| \Delta\left(f(\theta) r^{-n+2}\right)(\xi) d \xi \\
& -(2-n) \int_{S^{n-1}} f(\xi) d \xi
\end{aligned}
$$

Using the relation between the Fourier transform and differentiation, and applying the latter formula to the function $\Delta^{k-1}\left(f(\theta) r^{-n+2 k}\right)$ which is homogeneous of 
degree $-n+2$, we have

$$
\begin{gathered}
\left(f(\theta) r^{-n+2 k}\right)^{\wedge}(x)=(-1)^{k-1}\left(\Delta^{k-1}\left(f(\theta) r^{-n+2 k}\right)\right)^{\wedge}(x) \\
\quad=(-1)^{k} \int_{S^{n-1}} \ln |(x, \xi)| \Delta^{k}\left(f(\theta) r^{-n+2 k}\right)(\xi) d \xi \\
+(-1)^{k}(2-n) \int_{S^{n-1}} \Delta^{k-1}\left(f(\theta) r^{-n+2 k}\right)(\xi) d \xi .
\end{gathered}
$$

We will need the following spherical version of Parseval's formula; for the proof see [K4, Section 3.4].

Lemma 3.2. Let $K$ and $L$ be origin-symmetric infinitely smooth star bodies in $\mathbb{R}^{n}$ and $0<p<n$. Then

$$
\int_{S^{n-1}}\left(\|x\|_{K}^{-p}\right)^{\wedge}(\xi)\left(\|x\|_{L}^{-n+p}\right)^{\wedge}(\xi) d \xi=(2 \pi)^{n} \int_{S^{n-1}}\|x\|_{K}^{-p}\|x\|_{L}^{-n+p} d x .
$$

In what follows $C$ will always be a non-zero constant, not necessarily the same in different lines. We also use the notation $a(\epsilon) \sim b(\epsilon)$, meaning that $\lim _{\epsilon \rightarrow 0} a(\epsilon) / b(\epsilon)=1$.

Lemma 3.3. Let $p, q>0$ be integers, $p+q \leq n-2$.

(i) If $n-p-q-1$ is even, then for all $\xi \in S^{n-1}$

$$
\left(|x|_{2}^{-q}\|x\|_{E}^{-p}\right)^{\wedge}(\xi) \leq C \epsilon^{-n+p+q+1} .
$$

(ii) If $n-p-q-1$ is odd, then for every small $\alpha>0$ there exists a constant $C_{\alpha}$, such that for all $\xi \in S^{n-1}$,

$$
\left(|x|_{2}^{-q}\|x\|_{E}^{-p}\right)^{\wedge}(\xi) \leq C_{\alpha} \epsilon^{-n+p+q+1 /(1+\alpha)} .
$$

(iii) Moreover, in both cases,

$$
\left(|x|_{2}^{-q}\|x\|_{E}^{-p}\right)^{\wedge}\left(e_{n}\right) \sim C \epsilon^{-n+p+q+1} .
$$

Proof. (i) Let $n-p-q-1=2 k$. By Lemma 3.1 we have

$$
\left(|x|_{2}^{-q}\|x\|_{E}^{-p}\right)^{\wedge}(\xi)=\pi(-1)^{k} \int_{S^{n-1} \cap \xi^{\perp}} \Delta^{k}\left(|x|_{2}^{-q}\|x\|_{E}^{-p}\right) d x .
$$

Applying $\Delta$ under the integral, each time we get a factor of $1 / \epsilon^{2}$. This gives $\epsilon^{-2 k}=\epsilon^{-n+p+q+1}$. Finally, use the fact that $\|x\|_{E}^{-1} \leq 1$ for $x \in S^{n-1}$.

To prove (iii) for this case, we use (31) again. Keeping only the terms of the highest order of $\epsilon$, we get

$$
\left.\left(|x|_{2}^{-q}\|x\|_{E}^{-p}\right)^{\wedge}\left(e_{n}\right) \sim C \int_{S^{n-1} \cap e_{n}^{\perp}} \frac{\partial^{2 k}}{\partial x_{n}^{2 k}}\|x\|_{E}^{-p} d x \sim C \frac{\partial^{2 k}}{\partial x_{n}^{2 k}}\left(1+\frac{x_{n}^{2}}{\epsilon^{2}}\right)^{-p}\right|_{x_{n}=0}=C \epsilon^{-2 k} .
$$

(ii) Let $n-p-q-1=2 k-1$. By Lemma 3.1,

$$
\begin{aligned}
\left(|x|_{2}^{-q}\|x\|_{E}^{-p}\right)^{\wedge}(\xi) & =(-1)^{k} \int_{S^{n-1}} \ln |(x, \xi)| \Delta^{k}\left(|x|_{2}^{-q}\|x\|_{E}^{-p}\right) d x \\
& +(-1)^{k} \int_{S^{n-1}} \Delta^{k-1}\left(|x|_{2}^{-q}\|x\|_{E}^{-p}\right) d x
\end{aligned}
$$


Consider the first integral in (44). As before, applying the Laplacian $k$ times under the integral, we get a factor of $\epsilon^{-2 k}=\epsilon^{-n+p+q}$. Therefore we need to estimate terms of the following form:

$$
\int_{S^{n-1}} \ln |(\theta, \xi)|\|\theta\|_{E}^{-n+q} d \theta
$$

By Hölder's inequality, for a small $\alpha>0$,

$$
\begin{gathered}
\left|\int_{S^{n-1}} \ln \right|(\theta, \xi)\left|\|\theta\|_{E}^{-n+q} d \theta\right| \\
\leq\left(\int_{S^{n-1}}|\ln |(\theta, \xi)||^{(1+\alpha) / \alpha} d \theta\right)^{\alpha /(1+\alpha)}\left(\int_{S^{n-1}}\|\theta\|_{E}^{(-n+q)(1+\alpha)} d \theta\right)^{1 /(1+\alpha)} \\
=C_{\alpha}\left(\int_{S^{n-1}}\|\theta\|_{E}^{(-n+q)(1+\alpha)} d \theta\right)^{1 /(1+\alpha)} .
\end{gathered}
$$

Note that Parseval's formula (2) gives

$$
\begin{aligned}
& \int_{S^{n-1}}\|\theta\|_{E}^{(-n+q)(1+\alpha)} d \theta \\
& =(2 \pi)^{-n} \int_{S^{n-1}}\left(\|x\|_{E}^{(-n+q)(1+\alpha)}\right)^{\wedge}(\xi)\left(|x|_{2}^{n \alpha-q-q \alpha}\right)^{\wedge}(\xi) d \xi \\
& =C \epsilon \int_{S^{n-1}}\|\theta\|_{E^{*}}^{n \alpha-q-q \alpha} d \theta,
\end{aligned}
$$

where $E^{*}$ is the ellipsoid given by

$$
\|x\|_{E^{*}}=\left(x_{1}^{2}+\cdots+x_{n-1}^{2}+\epsilon^{2} x_{n}^{2}\right)^{1 / 2} .
$$

By the following elementary formula (see e.g. [G, p. 9])

$$
\int_{S^{n-1}} f((x, \theta)) d \theta=\left|S^{n-2}\right| \int_{-1}^{1}\left(1-t^{2}\right)^{(n-3) / 2} f(t) d t, \quad x \in S^{n-1},
$$

the integral in (5) equals

$$
\begin{gathered}
=C \epsilon \int_{-1}^{1}\left(1-t^{2}\right)^{(n-3) / 2}\left(1-t^{2}+\epsilon^{2} t^{2}\right)^{(n \alpha-q-q \alpha) / 2} d t \\
\sim C \epsilon \int_{-1}^{1}\left(1-t^{2}\right)^{(n-3+n \alpha-q-q \alpha) / 2} d t=C \epsilon .
\end{gathered}
$$

The latter integral converges if $(n-q)(1+\alpha)>1$, which is the case.

Therefore for all small $\alpha>0$,

$$
\left|\int_{S^{n-1}} \ln \right|(\theta, \xi)\left|\|\theta\|_{E}^{-n+q} d \theta\right| \leq C_{\alpha} \epsilon^{1 /(1+\alpha)}
$$

and hence the first integral in (44) can be bounded as follows:

$$
\left|\int_{S^{n-1}} \ln \right|(x, \xi)\left|\Delta^{k}\left(|x|_{2}^{-q}\|x\|_{E}^{-p}\right) d x\right| \leq C_{\alpha} \epsilon^{-n+p+q+1 /(1+\alpha)} .
$$

We use similar ideas to estimate the second integral in (4). Applying Parseval's formula two times and using the relation between the Fourier transform and 
differentiation, we get

$$
\begin{gathered}
\int_{S^{n-1}} \Delta^{k-1}\left(|x|_{2}^{-q}\|x\|_{E}^{-p}\right) d x=\int_{S^{n-1}} \Delta^{\frac{n-p-q-2}{2}}\left(|x|_{2}^{-q}\|x\|_{E}^{-p}\right)|x|_{2}^{-2} d x \\
=(2 \pi)^{-n} \int_{S^{n-1}}\left(\Delta^{\frac{n-p-q-2}{2}}\left(|x|_{2}^{-q}\|x\|_{E}^{-p}\right)\right)^{\wedge}(\theta)\left(|x|_{2}^{-2}\right)^{\wedge}(\theta) d \theta \\
=C \int_{S^{n-1}}\left(|x|_{2}^{-q}\|x\|_{E}^{-p}\right)^{\wedge}(\theta) d \theta=C \int_{S^{n-1}}\|x\|_{E}^{-p} d \theta=O(1),
\end{gathered}
$$

since $\|x\|_{E}^{-1} \leq 1$ on the sphere.

Combining the estimates for both terms in (4), one can see that

$$
\left(|x|_{2}^{-q}\|x\|_{E}^{-q}\right)^{\wedge}(\xi) \leq C_{\alpha} \epsilon^{-n+p+q+1 /(1+\alpha)} .
$$

Now we will show that almost the same degree of dependence is achieved when $\xi=e_{n}$. Indeed, using formula (4) with $\xi=e_{n}$ and dropping the second integral (which is small compared to the first integral), we get

$$
\left(|x|_{2}^{-p}\|x\|_{E}^{-q}\right)^{\wedge}\left(e_{n}\right) \sim(-1)^{k} \int_{S^{n-1}} \ln \left|x_{n}\right| \Delta^{k}\left(|x|_{2}^{-q}\|x\|_{E}^{-p}\right) d x .
$$

Formula (7) applied to the last integral gives

$$
=\left.2(-1)^{k} \int_{0}^{1} \ln x_{n} \cdot\left(1-x_{n}^{2}\right)^{\frac{n-3}{2}} \Delta^{k}\left(|x|_{2}^{-q}\|x\|_{E}^{-p}\right)\right|_{x_{1}^{2}+\cdots+x_{n-1}^{2}=1-x_{n}^{2}} d x_{n} .
$$

After the change of the variable $x_{n}=\epsilon \cdot z$, the latter equals

$$
\left.2(-1)^{k} \epsilon \int_{0}^{1 / \epsilon} \ln (\epsilon z) \cdot\left(1-\epsilon^{2} z^{2}\right)^{\frac{n-3}{2}} \Delta_{\epsilon}^{k}\left(\|x\|_{E^{*}}^{-q}|x|_{2}^{-p}\right)\right|_{x_{1}^{2}+\cdots+x_{n-1}^{2}=1-\epsilon^{2} z^{2}, x_{n}=z} d z,
$$

where $E^{*}$ is defined by (6), and

$$
\Delta_{\epsilon}=\frac{\partial^{2}}{\partial x_{1}^{2}}+\cdots+\frac{\partial^{2}}{\partial x_{n-1}^{2}}+\frac{1}{\epsilon^{2}} \frac{\partial^{2}}{\partial x_{n}^{2}} .
$$

Note that after writing $\ln (\epsilon z)=\ln \epsilon+\ln z$, we will have two integrals, the first being equal to

$$
\begin{aligned}
& \left.2(-1)^{k} \epsilon \ln \epsilon \int_{0}^{1 / \epsilon}\left(1-\epsilon^{2} z^{2}\right)^{\frac{n-3}{2}} \Delta_{\epsilon}^{k}\left(\|x\|_{E^{*}}^{-q}|x|_{2}^{-p}\right)\right|_{x_{1}^{2}+\cdots+x_{n-1}^{2}=1-\epsilon^{2} z^{2}, x_{n}=z} d z \\
= & (-1)^{k} \ln \epsilon \int_{S^{n-1}} \Delta^{k}\left(|x|_{2}^{-q}\|x\|_{E}^{-p}\right) d x=(-1)^{k} \ln \epsilon \int_{S^{n-1}} \Delta\left(\Delta^{k-1}\left(|x|_{2}^{-q}\|x\|_{E}^{-p}\right)\right) d x .
\end{aligned}
$$

Under the integral we have the Laplacian of a homogeneous function of degree $-n+2$ which equals the spherical Laplacian. Since the spherical Laplacian is a self-adjoint operator, the latter integral is equal to zero.

Therefore we only need to compute the order of the integral

$$
\left.2(-1)^{k} \epsilon \int_{0}^{1 / \epsilon} \ln z \cdot\left(1-\epsilon^{2} z^{2}\right)^{\frac{n-3}{2}} \Delta_{\epsilon}^{k}\left(\|x\|_{E^{*}}^{-q}|x|^{-p}\right)\right|_{x_{1}^{2}+\cdots+x_{n-1}^{2}=1-\epsilon^{2} z^{2}, x_{n}=z} d z .
$$

As before, the largest term is obtained when we apply $\frac{1}{\epsilon^{2}} \frac{\partial^{2}}{\partial x_{n}^{2}}$ to $|x|^{-p}$ successively $k$ times:

$$
\left.\sim 2(-1)^{k} \epsilon^{-2 k+1} \int_{0}^{1 / \epsilon} \ln z \cdot\left(1-\epsilon^{2} z^{2}\right)^{\frac{n-3}{2}} \frac{\partial^{2 k}}{\partial x_{n}^{2 k}}\left(|x|^{-p}\right)\right|_{x_{1}^{2}+\cdots+x_{n-1}^{2}=1-\epsilon^{2} z^{2}, x_{n}=z} d z .
$$


It is enough to show that

$$
\left.\int_{0}^{1 / \epsilon} \ln z \cdot\left(1-\epsilon^{2} z^{2}\right)^{\frac{n-3}{2}} \frac{\partial^{2 k}}{\partial x_{n}^{2 k}}\left(|x|^{-p}\right)\right|_{x_{1}^{2}+\cdots+x_{n-1}^{2}=1-\epsilon^{2} z^{2}, x_{n}=z} d z
$$

has a finite non-zero limit as $\epsilon \rightarrow 0$.

One can see that

$$
\begin{gathered}
\left.\lim _{\epsilon \rightarrow 0} \int_{0}^{1 / \epsilon} \ln z \cdot\left(1-\epsilon^{2} z^{2}\right)^{\frac{n-3}{2}} \frac{\partial^{2 k}}{\partial x_{n}^{2 k}}\left(|x|^{-p}\right)\right|_{x_{1}^{2}+\cdots+x_{n-1}^{2}=1-\epsilon^{2} z^{2}, x_{n}=z} d z \\
=\int_{0}^{\infty} \ln z \cdot \frac{\partial^{2 k}}{\partial z^{2 k}}\left(1+z^{2}\right)^{-p / 2} d z .
\end{gathered}
$$

To finish the proof, we need to show that the latter integral is not equal to zero. Let $P\left(z^{2}\right)=c_{0}+c_{1} z^{2}+\cdots+c_{k-1} z^{2 k-2}$ be the Taylor polynomial of $\left(1+z^{2}\right)^{-p / 2}$ at zero of order $2 k-2$. Then clearly,

$$
\int_{0}^{\infty} \ln z \frac{\partial^{2 k}}{\partial z^{2 k}}\left(1+z^{2}\right)^{-p / 2} d z=\int_{0}^{\infty} \ln z \frac{\partial^{2 k}}{\partial z^{2 k}}\left(\left(1+z^{2}\right)^{-p / 2}-P\left(z^{2}\right)\right) d z .
$$

After integration by parts $2 k$ times and the change of the variable $t=z^{2}$ the integral becomes

$$
\begin{aligned}
& =-(2 k-1) ! \int_{0}^{\infty} z^{-2 k}\left(\left(1+z^{2}\right)^{-p / 2}-P\left(z^{2}\right)\right) d z \\
& =-\frac{1}{2}(2 k-1) ! \int_{0}^{\infty} t^{-k-1 / 2}\left((1+t)^{-p / 2}-P(t)\right) d t .
\end{aligned}
$$

Using integration by parts in the opposite order and observing that $P(t)$ is the Taylor polynomial of $(1+t)^{-p / 2}$, we get

$$
\begin{aligned}
= & -\frac{1}{2} \frac{(2 k-1) !}{(1 / 2)(3 / 2) \cdots(k-1 / 2)} \int_{0}^{\infty} t^{-1 / 2} \frac{\partial^{k}}{\partial t^{k}}\left((1+t)^{-p / 2}-P(t)\right) d t \\
= & -\frac{1}{2} \frac{(2 k-1) !}{(1 / 2)(3 / 2) \cdots(k-1 / 2)} \int_{0}^{\infty} t^{-1 / 2} \frac{\partial^{k}}{\partial t^{k}}\left((1+t)^{-p / 2}\right) d t .
\end{aligned}
$$

The latter is clearly a non-zero constant.

Now we are ready to prove the main result of this section.

Theorem 3.4. For every $1 \leq k<l<n-3$ there exists an origin-symmetric convex body $K \in \mathbb{R}^{n}$ that does not belong to $\mathcal{I}_{k}$, but belongs to $\mathcal{I}_{l}$.

Proof. For a small $\epsilon>0$ define a body $K$ by

$$
\|x\|_{K}^{-1}=|x|_{2}^{-1}-\epsilon^{n-k-3 / 2}\|x\|_{E}^{-1}, \quad x \in \mathbb{R}^{n} \backslash\{0\},
$$

where $E$ is the ellipsoid with the norm

$$
\|x\|_{E}^{-1}=\left(x_{1}^{2}+\cdots+x_{n-1}^{2}+\frac{x_{n}^{2}}{\epsilon^{2}}\right)^{-1 / 2} .
$$

Convexity of $K$ follows along the lines of Lemma2.3. (One will need $\epsilon^{n-k-3 / 2-2}$ to be small, which is the case since $n-3>k$.)

Consider the $-l$ th power of the norm of $K$,

$$
\|x\|_{K}^{-l}=|x|_{2}^{-l}-\epsilon^{n-k-3 / 2} l|x|_{2}^{-l+1}\|x\|_{E}^{-1}+R(x),
$$


where $R(x)$ is the sum of the terms of the following form:

$$
\epsilon^{i(n-k-3 / 2)}|x|_{2}^{-l+i}\|x\|_{E}^{-i}, \quad i \geq 2 .
$$

Applying the Fourier transform, we have for all $\xi \in S^{n-1}$,

$$
\left(\|x\|_{K}^{-l}\right)^{\wedge}(\xi)=C(l, n)-\epsilon^{n-k-3 / 2} l\left(|x|_{2}^{-l+1}\|x\|_{E}^{-1}\right)^{\wedge}(\xi)+\widehat{R}(\xi) .
$$

By Lemma 3.3 the order of the second term is at most

$$
\epsilon^{n-k-3 / 2} \epsilon^{-n+l+1 /(1+\alpha)}=\epsilon^{l-k-1 / 2-\alpha /(1+\alpha)} \rightarrow 0, \text { as } \epsilon \rightarrow 0,
$$

if $\alpha$ is small enough. $\widehat{R}$ is even smaller, since it contains terms of the order $\epsilon^{i(n-k-3 / 2)} \epsilon^{-n+l+1 /(1+\alpha)}, i \geq 2$.

Therefore if $\epsilon$ is small, then $\left(\|x\|_{K}^{-l}\right)^{\wedge}(\xi) \geq 0$, and so $K \in \mathcal{I}_{l}$.

Now consider the $-k$ th power of the norm of $K$,

$$
\|x\|_{K}^{-k}=|x|_{2}^{-k}-\epsilon^{n-k-3 / 2} k|x|_{2}^{-k+1}\|x\|_{E}^{-1}+Q(x) .
$$

Computing the Fourier transform in the direction of $\xi=e_{n}$, we have

$$
\left(\|x\|_{K}^{-k}\right)^{\wedge}\left(e_{n}\right)=C(k, n)-\epsilon^{n-k-3 / 2} k\left(|x|_{2}^{-k+1}\|x\|_{E}^{-1}\right)^{\wedge}\left(e_{n}\right)+\widehat{Q}\left(e_{n}\right) .
$$

$\widehat{Q}\left(e_{n}\right)$ is small, since it has terms of order at most $\epsilon^{i(n-k-3 / 2)} \epsilon^{-n+k+1}, i \geq 2$. Therefore, we will pay attention only to the second term in (8). By Lemma 3.3

$$
\epsilon^{n-k-3 / 2}\left(|x|_{2}^{-k+1}\|x\|_{E}^{-1}\right)^{\wedge}\left(e_{n}\right) \sim C \epsilon^{n-k-3 / 2} \epsilon^{-n+k+1}=C \epsilon^{-1 / 2} .
$$

If we choose $\epsilon>0$ small enough so that the latter is greater than $C(k, n)$, then $\left(\|x\|_{K}^{-k}\right)^{\wedge}\left(e_{n}\right)<0$. So $K \notin \mathcal{I}_{k}$.

\section{ACKNOWLEDGEMENTS}

The author is thankful to Professors Alexander Koldobsky and Paul Goodey for many valuable suggestions.

\section{REFERENCES}

[BDK] J. Bretagnolle, D. Dacunha-Castelle and J. L. Krivine, Lois stables et espaces $L_{p}$, Ann. Inst. H. Poincaré Sect. B (N.S.) 2 (1965/66), 231-259. MR0203757 (34:3605)

[FGW] H. Fallert, P. Goodey and W. Weil, Spherical projections and centrally symmetric sets, Adv. Math. 129 (1997), 301-322. MR1462736 (98j:52005)

[G] H. Groemer, Geometric applications of Fourier series and spherical harmonics, Cambridge University Press, Cambridge and New York, 1996. MR1412143 (97j:52001)

[GS] I. M. Gelfand and G. E. Shilov, Generalized Functions, Vol. 1. Properties and Operations, Academic Press, New York and London, 1964. MR 0435831 (55:8786a)

[K1] A. Koldobsky, A Banach subspace of $L_{1 / 2}$ which does not embed in $L_{1}$ (isometric version), Proc. Amer. Math. Soc. 124 (1996), no. 1, 155-160. MR1285999 (96d:46026)

[K2] A. Koldobsky, Positive definite distributions and subspaces of $L_{-p}$ with applications to stable processes, Canad. Math. Bull. 42 (3) (1999), 344-353. MR1703694 (2001i:42014)

[K3] A. Koldobsky, A functional analytic approach to intersection bodies, Geom. Funct. Anal. 10 (2000), 1507-1526. MR:1810751 (2001m:52007)

[K4] A. Koldobsky, Fourier Analysis in Convex Geometry, Mathematical Surveys and Monographs, American Mathematical Society, vol. 116, Providence, RI, 2005. MR.2132704 (2006a:42007)

[KK] N. Kalton, A. Koldobsky, Banach spaces embedding isometrically into $L_{p}$ when $0<p<1$, Proc. Amer. Math. Soc. 132 (2004), no. 1, 67-76. MR2021249 (2004h:46009)

[L] E. Lutwak, Intersection bodies and dual mixed volumes, Advances in Math. 71 (1988), 232-261. MR 963487 (90a:52023)

[M1] E. Milman, Generalized intersection bodies, J. Funct. Anal. 240 (2006), no. 2, 530-567. MR2261694 (2007h:52007) 
[M2] E. Milman, Generalized intersection bodies are not equivalent, preprint, arXiv:math/0701779.

[N] A. Neyman, Representation of $L_{p}$-norms and isometric embedding in $L_{p}$-spaces, Israel J. Math. 48 (1984), 129-138. MR770695 (86g:46033)

[S] J. Schlieper, A note on k-intersection bodies, Proc. Amer. Math. Soc. 135 (2007), 20812088. MR2299484

[W] W. Weil, Zonoide und verwandte Klassen konvexer Körper, Monatsh. Math. 94 (1982), 73-84. MR670016 (84e:52008)

[Y] M. Yaskina, Non-intersection bodies, all of whose central sections are intersection bodies, Proc. Amer. Math. Soc. 135 (2007), 851-860. MR2262882 (2007i:52007)

[Z] Gaoyong Zhang, Sections of convex bodies, Amer. J. Math. 118 (1996), 319-340. MR.1385280 (97f:52015)

Department of Mathematics, University of Oklahoma, Norman, Oklahoma 73019

E-mail address: vyaskin@math.ou.edu 